\title{
PROPIEDADES FÍSICO MECÁNICAS DE BLOQUES H10 FABRICADOS EN EL ÁREA METROPOLITANA DE CÚCUTA
}

\section{MECHANICAL/PHYSICAL PROPERTIES OF H10 HOLLOW BRICKS MANUFACTURED IN THE METROPOLITAN AREA OF CÚCUTA}

\author{
Sandra Milena Rozo Rincón', Jorge Sánchez Molina², Diana Carolina Álvarez Rozo³
}

Fecha de recepción: 23 de julio de 2013

Fecha de aceptación: 10 de marzo de 2014

Referencia: S.M. Rozo Rincón, J. Sánchez Molina, D.C. Álvarez Rozo. (2014). Propiedades físico mecánicas de bloques H10 fabricados en el área Metropolitana de Cúcuta. Ciencia e Ingeniería Neogranadina, 24 (1), pp. 67- 78.

\section{RESUMEN}

En este artículo se presentan los resultados de las propiedades físicas y mecánicas de dos tipos de bloques $\mathrm{H} 10$ (bloque seis huecos cuadrado, bloque ocho huecos mixto) fabricados en Cúcuta y su área metropolitana. Se visitaron diferentes empresas en las que se recolectaron muestras de cada tipo de bloque fabricado. Se realizaron ensayos físicos como absorción de agua, densidad, dimensiones y resistencia a la compresión en el laboratorio del Centro de Investigación de Materiales Cerámicos siguiendo los lineamientos de la norma NTC 4017. Los resultados se compararon con los datos establecidos en la norma NTC 4205, y se logra determinar que los diferentes bloques poseen propiedades semejantes, las cuales permiten su uso como productos de mampostería no estructural para uso interior o exterior.

Palabras clave: bloque cerámico, compresión, NTC 4017

\section{ABSTRACT}

In this article, we present the physical and mechanical properties of two types of H10 hollow bricks (six hollow square brick, eight holes mixed brick) manufactured in Cucuta and its metropolitan area. Different companies were visited and samples were collected from each type of manufactured hollow brick. Physical tests, such as water absorption, density, dimensions, and compressive strength were performed in the Research Center of

\footnotetext{
1. Ing. de Producción Industrial. Estudiante de maestría en Ciencia y tecnología de Materiales, sandrarozo85@hotmail.com, Centro de Investigación de Materiales Cerámicos, Universidad Francisco de Paula Santander, Cúcuta, Colombia.

2. Ing. Químico, M.Sc Gerencia de empresas mención Industria, j_sanchezmolina@yahoo.es, Profesor asociado, Facultad de Ciencias Básicas, Investigador Grupo GITEC, Universidad Francisco de Paula Santander, Cúcuta, Colombia.

3. Ing. Industrial. Joven Investigadora UFPS-COLCIENCIAS, d_alvarezrozo@yahoo.com, Investigadora Grupo GITEC, Centro de Investigación de Materiales Cerámicos, Universidad Francisco de Paula Santander, Cúcuta, Colombia.
} 
Ceramic Materials Laboratory following the guidelines of the NTC 4017. The results were compared to the data provided by the standard NTC 4205. We were able to determine that the different hollow bricks have similar properties that make its use as nonstructural masonry products for indoor and outdoor use possible.

Keywords: ceramic block, compression, NTC 4017.

\section{INTRODUCCIÓN.}

En Cúcuta y su área metropolitana se fabrican diferentes productos para construcción, dentro de los cuales sobresale el bloque $\mathrm{H} 10$, en presentaciones como seis huecos cuadrado y ocho huecos mixto [1]. Estos productos se emplean como mampostería en obras civiles. Su función consiste en soportar cargas uniaxiales de fuerza perpendicular al área portante [2]. Este tipo de productos se encuentra expuesto a humedad del interior o exterior por lo cual debe poseer un bajo índice de absorción de agua, que evite dilataciones, fisuras y desprendimiento del mortero.

La calidad de los productos de construcción es un factor de importancia para las obras civiles, que cada día son más exigentes para garantizar edificaciones sismoresistentes [3].
Esta investigación tuvo como objetivo determinar las propiedades físicas y mecánicas de los bloques H10 fabricados en Cúcuta y su área metropolitana, siguiendo los parámetros de la norma NTC 4017. Esto con el propósito de lograr identificar la variación en las dimensiones de los productos fabricados y el cumplimiento de propiedades establecidas en la norma NTC 4205-2, como espesor de tabiques, paredes, absorción de agua y resistencia mecánica a la compresión.

\section{MATERIALES Y MÉTODOS}

Las características morfológicas 0 dimensionales del bloque $\mathrm{H} 10$ son largo, ancho, altura del piso a la pieza, espesor y peso, relacionadas en la Figura 1.

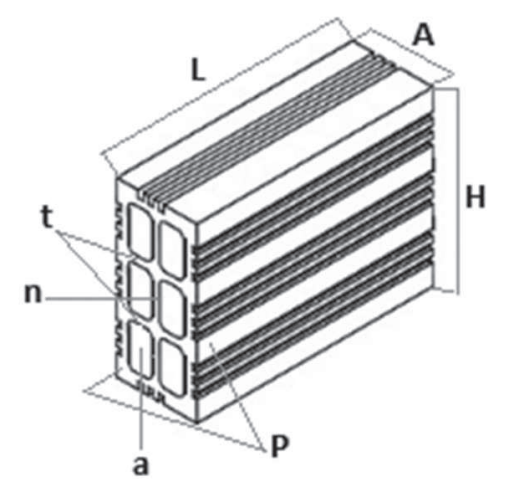

Figura 1. Dimensiones del bloque $\mathrm{H} 10$.

Fuente: Asociación española de fabricantes de ladrillos y tejas de arcilla cocida HISPALYT. 
Donde:

$$
\begin{aligned}
& \mathrm{L}=\operatorname{largo}(\mathrm{cm}) \\
& \mathrm{A}=\operatorname{ancho}(\mathrm{cm}) \\
& \mathrm{H}=\text { alto }(\mathrm{cm}) \\
& \mathrm{P}=\text { espesor más pequeño de las paredes }(\mathrm{mm}) \\
& \mathrm{T}=\text { espesor más pequeño de los tabiques }(\mathrm{mm}) \\
& \mathrm{N}=\text { espesor del nervio }(\mathrm{mm})
\end{aligned}
$$

En este estudio se han tomado en cuenta las empresas de Cúcuta y su área metropolitana. Según información de la Asociación de Industriales de la Arcilla, Induarcilla, a la fecha se encuentran vigentes 90 empresas dedicadas a la fabricación de productos cerámicos [5].

La muestra objeto de estudio se identificó mediante muestreo probabilístico para poblaciones finitas [6], mediante la siguiente expresión:

$$
x=\frac{\mathrm{N} * Z^{2} * p * q}{d^{2} *(\mathrm{~N}-1)+Z^{2} * p * q}
$$

Donde:

$\mathrm{N}=$ Total de la población

Z= Nivel de confianza, 1,96 para un nivel de seguridad del $95 \%$

$\mathrm{p}=$ Probabilidad de que el evento ocurra $(0,05)$ $q=1-p(0,95)$

$\mathrm{d}=$ grado de precisión -margen de error, para este caso 0,05

La metodología utilizada para determinar las propiedades físicas y mecánicas de los ladrillos fue tomada de la NTC 4017, métodos para muestreo y ensayos de unidades de mampostería y otros productos de arcilla [7], en donde se exponen los procedimientos de selección y preparación de la muestra.

La resistencia a la compresión de ladrillo de arcilla, $f^{-}{ }_{c u}$, se usa como control de calidad en la elaboración (dosificación de los materiales, temperatura y tiempo por quema), para conocer la calidad de los materiales y la materia prima utilizados en la fabricación de ladrillo y para encontrar la resistencia a la compresión de la mampostería, $\mathrm{f}_{\mathrm{m}}^{-}$, a partir de fórmulas que relacionan las propiedades de la unidad y los morteros [8].

Para la realización de ensayos físicos de los bloques se secaron las muestras a $110^{\circ} \mathrm{C}$ en la estufa de secado marca Gabrielli, hasta alcanzar el peso constante. Los ensayos se realizaron para 10 muestras de bloque de cada una de las empresas. Se tomaron las dimensiones de los bloques H10 (longitud y ancho) con el fin de observar la variabilidad entre las diferentes empresas para lo cual se empleó el calibrador pie de rey marca Mitutoyo. Igualmente, se tomaron los espesores de las paredes y tabiques. Los resultados fueron comparados con los datos establecidos en la norma NTC 4205. Se tomó el peso de cada una de las muestras en la balanza analítica con 0,1 de precisión.

En la caracterización de bloques según los lineamientos de la norma NTC 4017 se realizaron los ensayos de absorción de agua y resistencia mecánica a la compresión para cinco muestras de cada empresa, los resultados fueron comparados con los datos establecidos en la norma NTC 4205. 
Los resultados de los ensayos realizados a los bloques $\mathrm{H} 10$ seis huecos fueron comparados con los de bloques ocho huecos. Estos valores fueron analizados con la norma NTC 4205 para evaluar la conformidad de los productos fabricados actualmente en el área metropolitana de Cúcuta.

\section{RESULTADOS Y ANÁLISIS}

\subsection{CARACTERISTICAS GENERALES DE LA PRODUCCIÓN DE BLOQUES H10 EN EL ÁREA METROPOLITANA DE CÚCUTA}

Se encuestaron cuarenta empresas, de las cuales se determinó que treinta y tres de ellas fabrican bloque H10. Las principales características de producción se presentan en la Tabla 1.

Tabla 1. Características de producción del bloque H10.

\begin{tabular}{|c|c|}
\hline & DESCRIPCIÓN \\
\hline $\begin{array}{l}\text { Productos } \\
\text { fabricados }\end{array}$ & $\begin{array}{l}\text { De las empresas estudiadas, el } 77 \% \text { fabrica bloque } 6 \text { huecos cuadrado, que } \\
\text { es el de mayor producción, seguido por el bloque mixto } 8 \text { huecos con el } 13 \% \\
\text { de participación. El } 10 \% \text { restante fabrica bloque } 8 \text { huecos redondo y } 6 \text { huecos } \\
\text { vitrificado. En el estudio se recolectaron muestras del bloque } 6 \text { huecos } \\
\text { cuadrado y el bloque mixto } 8 \text { huecos, ya que son de fabricación continua en } \\
\text { las empresas estudiadas. }\end{array}$ \\
\hline $\begin{array}{l}\text { Materias } \\
\text { primas } \\
\text { empleadas }\end{array}$ & $\begin{array}{l}\text { La principal materia prima utilizada en la fabricación del bloque es la arcilla } \\
\text { roja, debido a que la región cuenta con abundantes yacimientos de este tipo } \\
\text { [9]. El } 59 \% \text { de las empresas estudiadas no realiza mezclas arcillosas para la } \\
\text { elaboración de dicho producto, el } 41 \% \text { restante sí realiza mezclas, en las que } \\
\text { se incluye la adición de diversos componentes como arena silícea, arcilla } \\
\text { amarilla, chamota. Algunas empresas también incluyen la adición de arcilla gris } \\
\text { y caliza. }\end{array}$ \\
\hline $\begin{array}{l}\text { Controles } \\
\text { a las } \\
\text { materias } \\
\text { primas }\end{array}$ & $\begin{array}{l}\text { Tan solo el } 34 \% \text { de las empresas estudiadas realiza algún control a la materia } \\
\text { prima. Entre los ensayos más representativos se tienen: determinación del } \\
\text { tamaño de partícula, que permite controlar la distribución granulométrica de las } \\
\text { materias primas empleadas; e índice de plasticidad, que permite identificar la } \\
\text { cantidad de agua adecuada para la humectación de la materia prima [10]. }\end{array}$ \\
\hline $\begin{array}{l}\text { Controles } \\
\text { en el } \\
\text { proceso } \\
\text { productivo }\end{array}$ & $\begin{array}{l}\text { En las etapas del proceso productivo es importante realizar controles con el fin } \\
\text { de garantizar la calidad de los productos. El } 97 \% \text { de las empresas que } \\
\text { fabrican bloque tienen dentro de su política de calidad realizar controles sobre } \\
\text { el producto en proceso; los ensayos que se realizan con mayor frecuencia son } \\
\text { la identificación de contracciones en secado, dosificación de materia prima y, } \\
\text { en menor importancia, tiempo de cocción. }\end{array}$ \\
\hline $\begin{array}{l}\text { Controles } \\
\text { sobre la } \\
\text { materia } \\
\text { prima. }\end{array}$ & $\begin{array}{l}\text { El control realizado sobre el producto terminado es de gran importancia, ya } \\
\text { que permite clasificar si el producto es de primera, segunda o tercera clase, así } \\
\text { como garantizar la reproducibilidad de los productos que se ofrecen en el } \\
\text { mercado [11]. Tan sólo el } 11 \% \text { de las empresas que fabrican bloque realizan } \\
\text { control al producto terminado sobre las dimensiones de sus productos, masa } \\
\text { del bloque, porcentaje de absorción de agua y resistencia a la compresión. }\end{array}$ \\
\hline
\end{tabular}




\subsection{CARACTERIZACIÓN FÍSICA DE LAS MUESTRAS.}

A continuación se presentan los resultados para medición de largo, ancho, espesores de pared y tabiques.

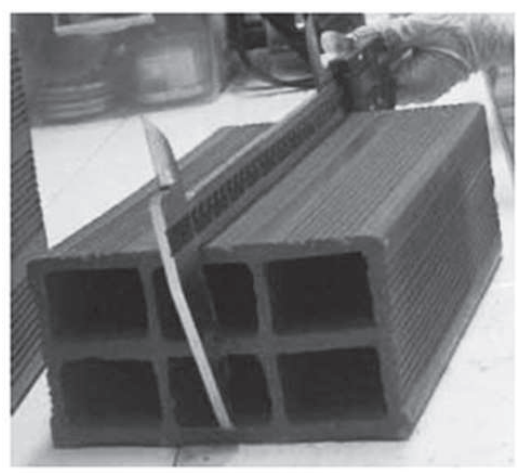

Figura 2. Medición de dimensiones de los productos de mampostería de perforación horizontal.
Los productos de mampostería de perforación horizontal no presentan uniformidad en el largo en las diferentes empresas estudiadas (Figura 3). Esto se debe a que no existe unificación de este parámetro en las diferentes empresas. Esta medida depende del corte una vez sale el producto de la extrusora, además de un buen cálculo de las contracciones que sufre este tipo de producto en el secado y la cocción. Para el bloque seis huecos, los valores oscilan entre 28,00 y $30,50 \mathrm{~cm}$, con un promedio de 29,48 $\mathrm{cm}$, desviación estándar de $\pm 0,74$. Para el caso del bloque ochos huecos mixto el valor del largo oscila entre 29,13 y 30,95 $\mathrm{cm}$, con un promedio de 30,25 y una desviación estándar de $\pm 0,73$.

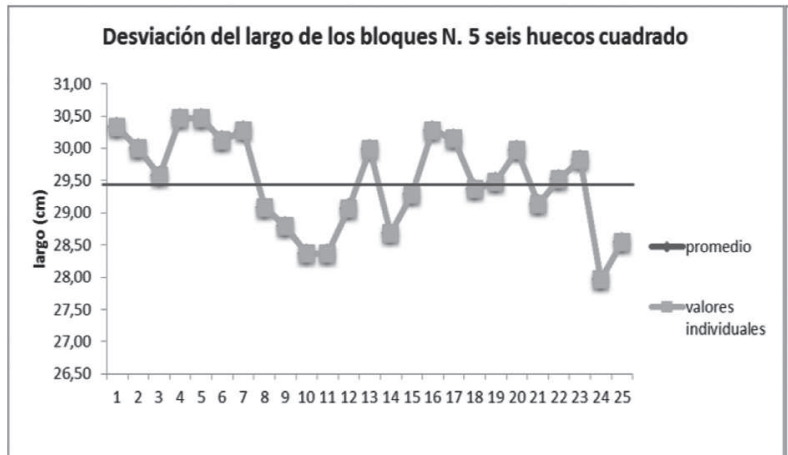

(a)

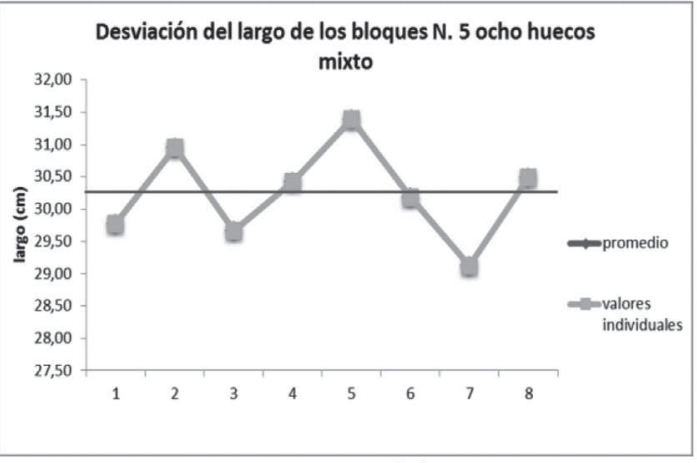

(b)

Figura 3. (a) Largo de bloques H10 seis huecos cuadrados.

(b) Largo de los bloques H10 ocho huecos mixtos. 


\section{UNIVERSIDAD MILITAR NUEVA GRANADA - CIENCIA E INGENIERÍA NEOGRANADINA}

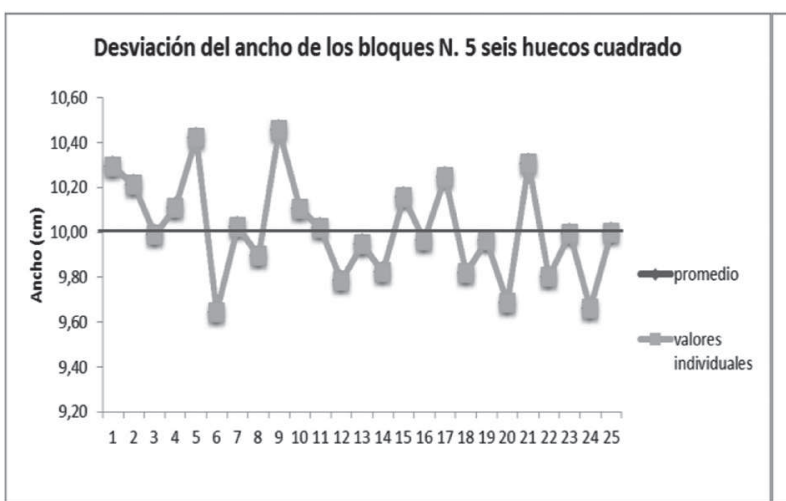

(a)

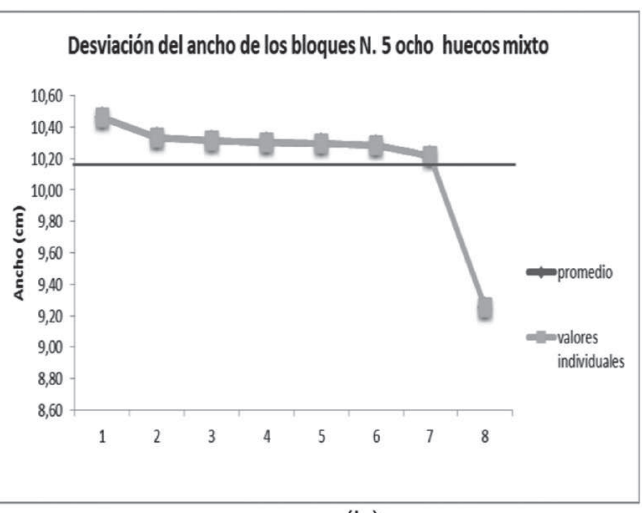

(b)

Figura 4. (a) Ancho de bloques $\mathrm{H} 10$ seis huecos cuadrado.

(b) Ancho de los bloques H10 ocho huecos mixto.

La variación del ancho de los productos de mampostería de perforación horizontal está relacionada con el ancho del molde. El ancho de los productos extruidos es el valor inicial, el cual se ve afectado posteriormente por las contracciones que sufre durante el secado y la cocción. Esta contracción es característica de cada materia prima usada en el proceso, razón por la cual el molde debe ser diseñado teniendo en cuenta este parámetro. Los valores del ancho de los bloques seis huecos se encuentran entre 9,64-10,46 cm, un promedio de $10,01 \mathrm{~cm}$ $\pm 0,23$. Los valores del ancho de los bloques ocho huecos se encuentran entre 10,22$10,46 \mathrm{~cm}$, un promedio de 10,18 $\pm 0,07$. Para el cálculo del promedio se tomaron en cuenta los datos de las 7 empresas, pues el valor de la empresa $8(9,25 \mathrm{~cm})$ se aparta claramente del resto de las instalaciones, lo que desvirtuaría dicho valor.

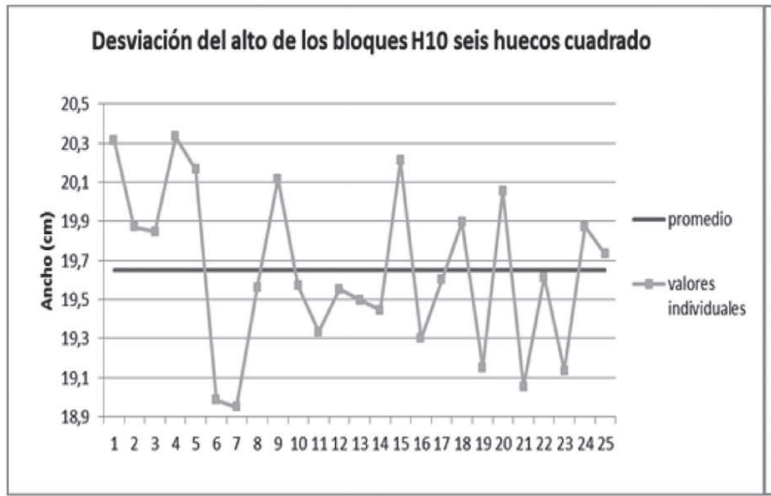

(a)

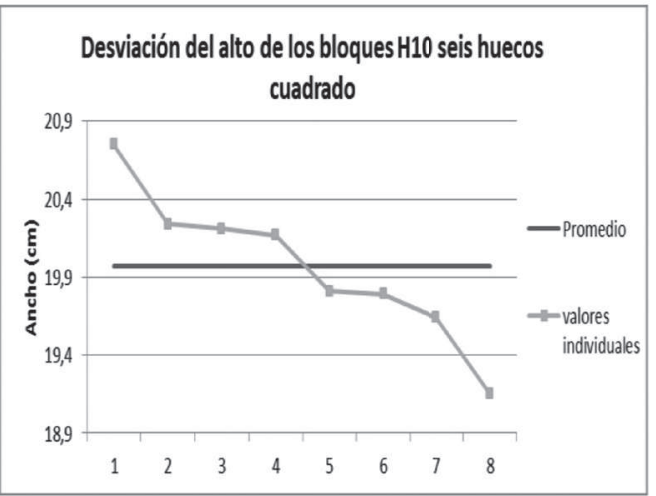

(b)

Figura 5. (a) Alto de bloques H10 seis huecos cuadrado.

(b) Alto de los bloques H10 ocho huecos mixto. 
El alto de los productos varía en un pequeño rango en los bloques $\mathrm{H} 10$ seis huecos entre $19,05-20,31 \mathrm{~cm}$, con un promedio de 19,65 $\pm 0,42$. El alto de los bloques $\mathrm{H} 10$ ocho huecos mixto se encuentra entre 19,15 $20,75 \mathrm{~cm}$, con un promedio de 19,97 $\pm 0,48$. Esta medida depende del molde usado en la extrusora y de las contracciones de la materia prima.

\subsubsection{Medición de espesor de tabiques y paredes}

En la Figura 6 se presentan los tabiques y paredes de los bloques. Los resultados se presentan en las Figuras 4 y 5.

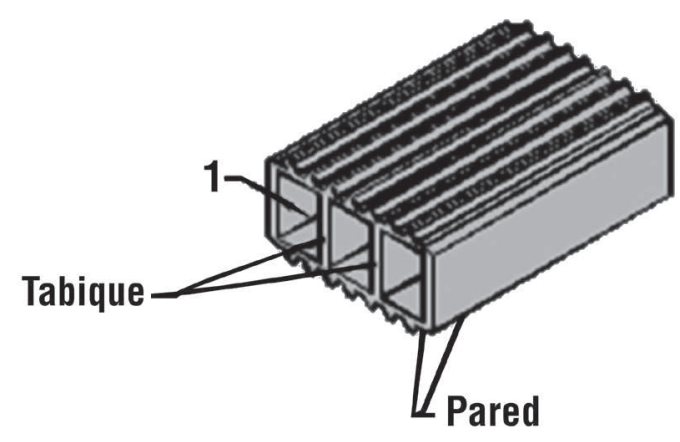

Figura 6. Tabiques y paredes de productos de mampostería de perforación horizontal

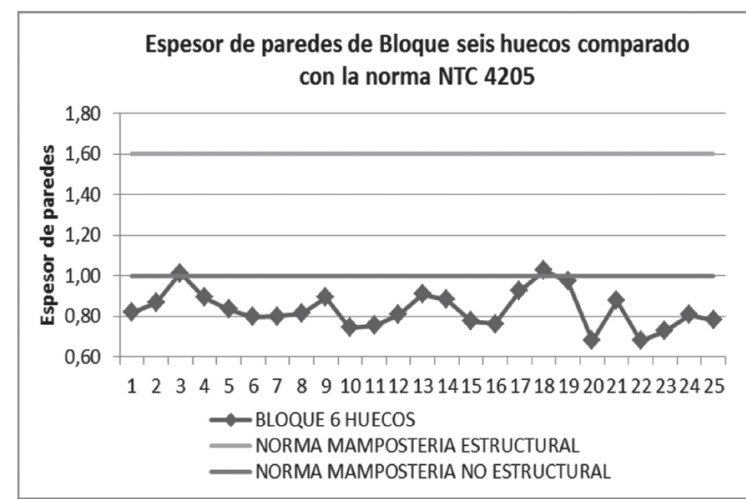

(a)

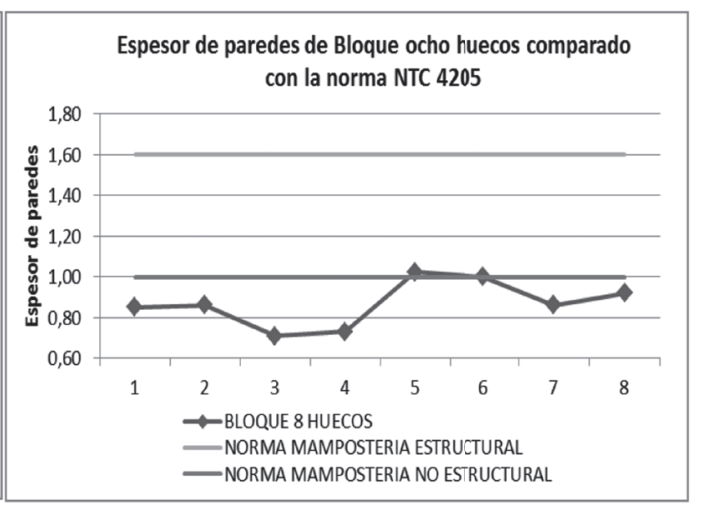

(b)

Figura 7. (a) Espesor de paredes de bloques $\mathrm{H} 10$ seis huecos cuadrado.

(b) Espesor de paredes de los bloques H10 ocho huecos mixto.

El espesor mínimo de las paredes según la norma NTC 4205 debe ser de 1,60 $\mathrm{cm}$ para productos de mampostería estructural y $1,00 \mathrm{~cm}$ para mampostería no estructural. En la Figura 6 se observa, mayoritariamente, que los bloques $\mathrm{H} 10$ seis huecos y ocho huecos fabricados en la región se encuentran por debajo de la norma para ambos casos. 


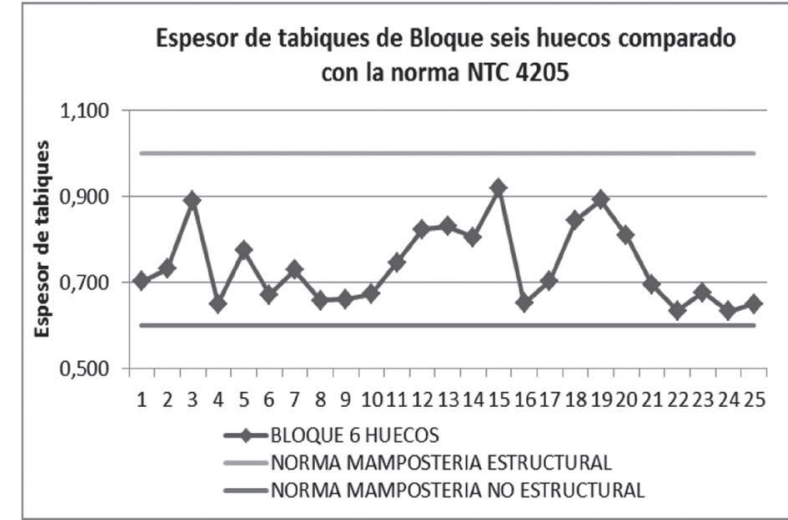

(a)

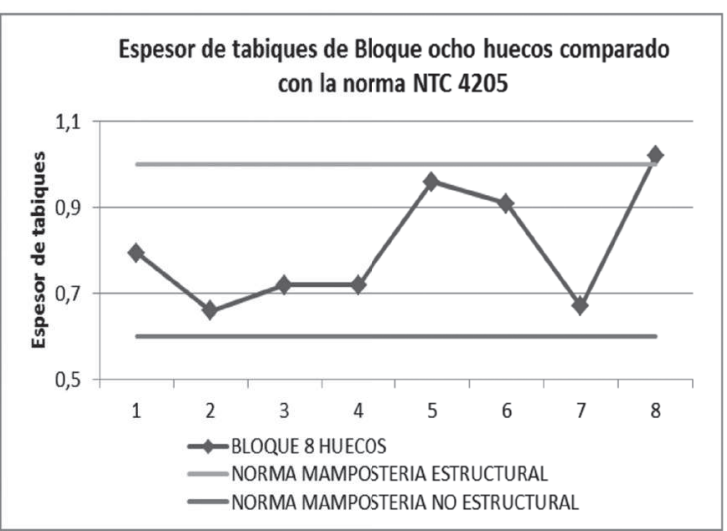

(b)

Figura 8. (a) Espesor de tabiques de bloques H10 seis huecos cuadrado.

(b) Espesor de tabiques de los bloques H10 ocho huecos mixto.

El espesor mínimo de los tabiques según la se observa que los bloques $\mathrm{H} 10$ seis huecos norma NTC 4205 debe ser de 1,00 y 0,60 y ocho hueco mixto están cumpliendo con cm para mampostería estructural y no el espesor de tabiques para productos de estructural respectivamente. En la figura 7 mamposteria no estructural.

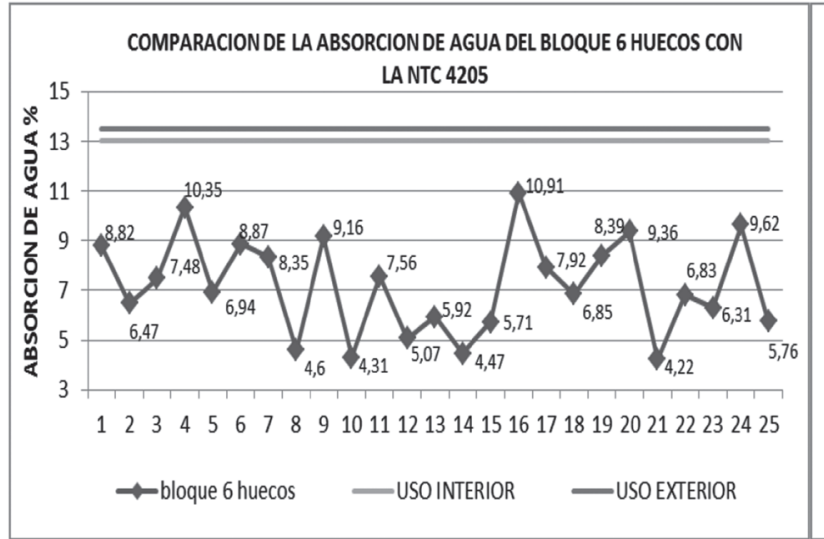

(a)

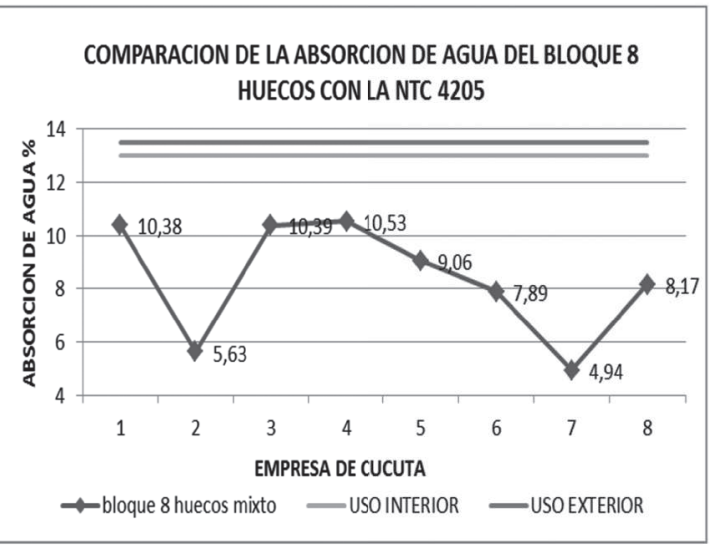

(b)

Figura 9. (a) Absorción de agua de bloques H10 seis huecos cuadrado.

(b) Absorción de agua de los bloques H10 ocho huecos mixto. 
La absorción de agua tiene una relación directa con la porosidad, propiedades mecánicas, densidad aparente y compactación de un producto cerámico. Se considera un parámetro importante, ya que refleja la calidad del producto y, por esta razón, es uno de los ensayos realizados con mayor frecuencia [12]. Menores valores de absorción de agua indican que el producto tiene menor porosidad abierta, lo cual representa una ventaja pues el producto tiene menor capacidad de absorber agua del ambiente. La baja porosidad tiene relación con el grado de compactación de la mezcla arcillosa durante su fabricación [13].

Los valores máximos de absorción de agua según la norma NTC 4205 deben ser de 13 \% y 13,5 \% según sean para uso interior y exterior respectivamente. Estos valores son de igual magnitud para mampostería estructural y no estructural [14]. En la Figura 9 se observa que los bloques seis huecos y ocho huecos se encuentran muy por debajo de la norma, razón por la cual este tipo de productos puede ser empleado tanto para interiores como para exteriores.

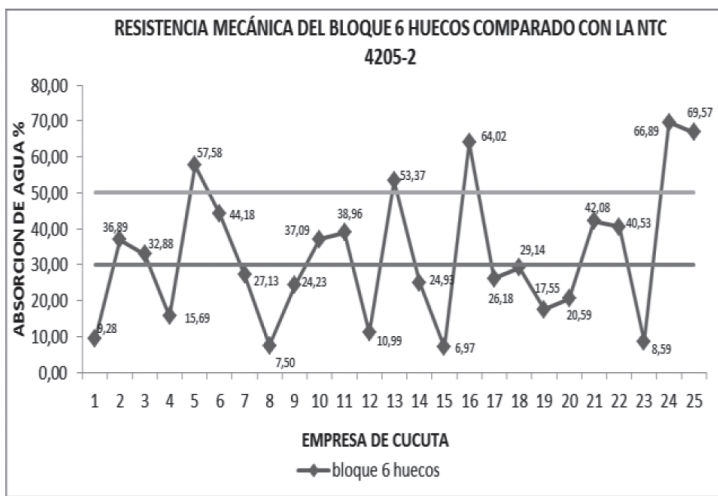

(a)

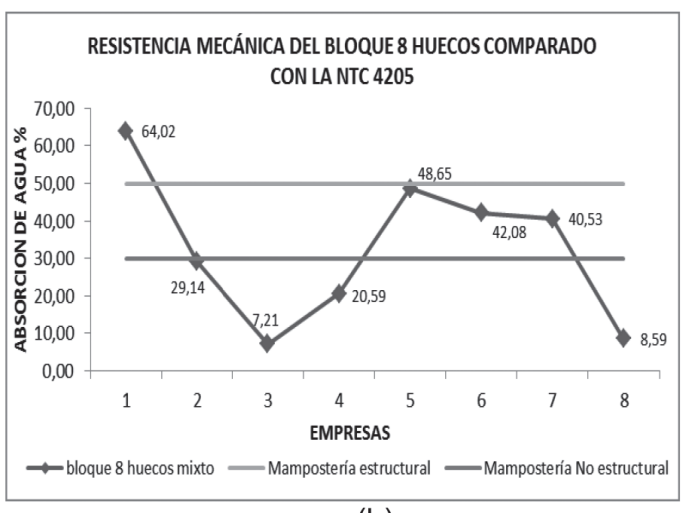

(b)

Figura 10. (a) Resistencia mecánica a la compresión de bloques H10 seis huecos cuadrado. (b) Resistencia mecánica a la compresión de bloques H10 ocho huecos mixto.

Los valores mínimos de resistencia a la compresión se encuentran entre 30 y 50 $\mathrm{kgf} / \mathrm{cm}^{2}$ para mampostería estructural y no estructural respectivamente. El $48 \%$ de las empresas que fabrican bloques $\mathrm{H} 10$ seis huecos cumplen con los valores de resistencia mecánica establecidos en la norma NTC 4205, mientras que el $52 \%$ restante no los cumple. Para el caso de los bloques H10 ocho huecos mixto el $50 \%$ de las empresas cumplen con los parámetros de la norma.

Las discrepancias en los valores de la resistencia mecánica pueden ser ocasionadas por defectos como grietas internas o externas que hacen que el producto resista menos carga antes de la fractura. Estos defectos dependen de los controles que se hagan en las empresas y de la manipulación que tenga el producto en las diferentes etapas del proceso [15]. 


\subsection{RELACIÓN DE VARIABLES}

En la figura 11 se presenta la relación existente entre la absorción de agua y la resistencia mecánica a la compresión de bloques 8 huecos mixto. Se observa que a medida que incrementa la absorción de agua, se reduce la resistencia mecánica de los bloques 8 huecos mixto. Esto puede ser ocasionado por la alta porosidad y tensiones internas que hacen más frágiles los productos.
Las demás variables no lograron ser interrelacionadas debido a que las muestras son de diferentes empresas, en donde se emplean materias primas y formulaciones de pasta distintas, las cuales presentan un comportamiento característico durante el proceso productivo y confieren a los productos propiedades variables.

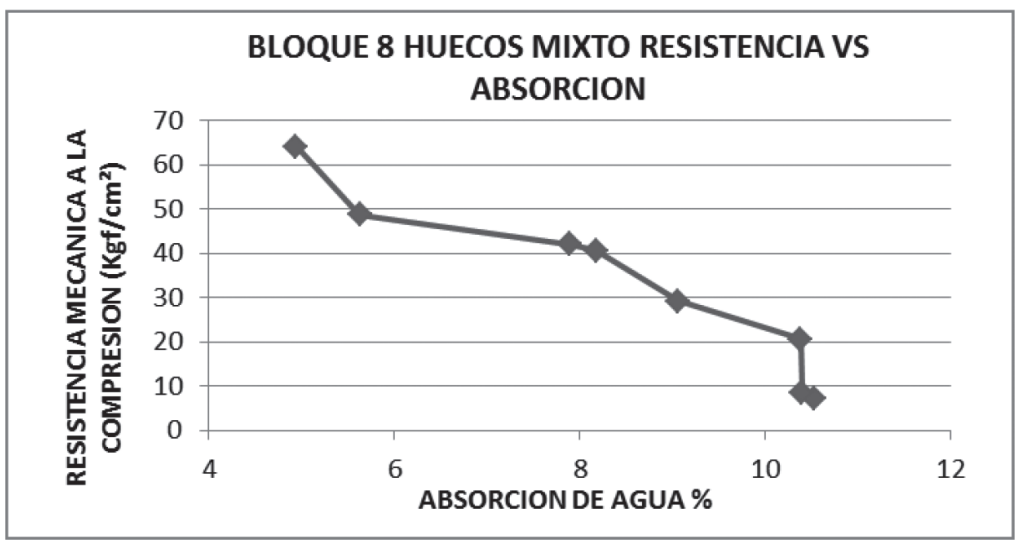

Figura 11. Relación de variables del bloque 8 huecos mixto.

\section{CONCLUSIONES}

Los resultados obtenidos permiten identificar que los bloques H10 fabricados en el área metropolitana de Cúcuta se deben estandarizar en cuanto a la medida de longitud, ancho y largo, ya que presenta variabilidad entre las diferentes empresas que los fabrican.

El espesor de las paredes de los bloques H10 no cumple con los parámetros establecidos en la norma NTC 4205, debido a que se encuentran por debajo del valor mínimo requerido, mientras que el espesor de los tabiques y el nervio se encuentran por encima del valor mínimo establecido en la norma.

La norma NTC 4205 establece que para productos cerámicos de mampostería no estructural el porcentaje la absorción de agua debe ser máximo de $13.5 \%$. Como resultado de los datos obtenidos se pude concluir que los bloques seis huecos y ocho huecos fabricados en la región cumplen 
con lo estipulado por la norma, por lo que pueden ser empleados tanto para interiores como para exteriores.

La resistencia mecánica a la compresión depende directamente del porcentaje de absorción de agua que tengan las piezas. Los valores de resistencia entre los bloques seis huecos y ocho huecos son valores de magnitudes similares por lo cual se puede decir que estos bloques tienen una resistencia mecánica semejante para emplearlos como mampostería no estructural

Los bloques $\mathrm{H} 10$ de seis huecos cuadrado y ocho huecos mixto presentan propiedades similares. En las condiciones actuales de fabricación este producto puede ser empleado como mampostería de tipo no estructural.

\section{AGRADECIMIENTOS}

Los autores expresan sus agradecimientos a la Universidad Francisco de Paula Santander, de manera especial al Centro de Investigación de Materiales Cerámicos y a las empresas que fueron objeto de estudio, por su aporte en la ejecución de esta investigación.

\section{REFERENCIAS}

[1] Sánchez Molina, J. (2010). Estrategias para la competitividad del clúster de la cerámica del área metropolitana de Cúcuta (Tesis de maestría inédita). Universidad Nacional Experimental del Táchira, San Cristóbal, República Bolivariana de Venezuela.
[2] López, J. (1998). Cálculo del comportamiento de la mampostería mediante elementos finitos. Recuperado en febrero de 2013, de http://www.cimne.com/personales/ eo/publicaciones/files/M46.pdf.

[3] Peña Rodríguez, G. (2008). Efecto de la concentración de poliestireno expandido en la conductividad térmica efectiva de bloques de termoarcilla. Revista colombiana de física, 40(2).

[4] Asociación española de fabricantes de ladrillos y tejas de arcilla cocida HISPALYT. (2008). Control de recepción en obra de productos de cerámica estructural utilizados en la construcción. Madrid, España: HISPALYT Asociación española de fabricantes de ladrillos y tejas de arcilla cocida.

[5] Induarcilla. (s.f.). Presentación del Centro de Investigación de Materiales Cerámicos Cimac. Recuperado el 15 de noviembre de 2011, de http:// www.induarcilla.com/cimac/

[6] Weiers, Ronald M. (1986). Investigación de mercados. México. Editorial Prentice HallHispanoamericano. Página 123.

[7] Instituto Colombiano de Normas Técnicas y Certificación ICONTEC NTC 4017. (2005). Métodos para muestreo y ensayos de unidades de mampostería y otros productos 
de arcilla. 1ra actualización. Bogotá, Colombia: ICONTEC.

[8] Takeuchi, C. (2007). Comportamiento en la mampostería estructural. Bogotá, Colombia: Universidad Nacional de Colombia.

[9] Sánchez Polentino, Y. \& Mora Sepúlveda, M. (2012). Estudio comparativo de las propiedades físico mecánicas de los diferentes tipos de bloques $N^{\circ} 5$ fabricados en el área metropolitana de Cúcuta (Trabajo de grado inédito). Universidad Francisco de Paula Santander, Cúcuta, Colombia.

[10] Grupo de Investigación en Tecnología Cerámica GITEC. (2011). Evaluación de los cambios en las propiedades térmicas y mecánicas del bloque $N^{\circ} 5$ fabricado en la región, según las mezclas entre arcilla y residuos industriales. Cúcuta, Colombia: Universidad Francisco de Paula Santander

[11] Carrillo Neira, L. y Leal Cárdenas, D. (2012). Estudio comparativo de teja curva y bloque $N^{\circ} 5$ de arcilla bajo normas nacionales e internacionales para el sector cerámico del área metropolitana de Cúcuta (Trabajo académico inédito). Universidad Francisco de Paula Santander, Cúcuta, Colombia.

[12] Díaz García, I. (2012). Estandarización de tejas y bloques del sector cerámico en Norte de Santander. Cúcuta,
Colombia: Convenio Universidad Francisco de Paula Santander, Cámara de Comercio de Cúcuta e Induarcilla.

[13] Sánchez Molina, J. \& Monroy Sepúlveda, R. (2004). Estudio para producir termoarcilla con mezclas de arcilla, poliestireno y cenizas. Cúcuta, Colombia: Universidad Francisco de Paula Santander.

[14] Instituto Colombiano de Normas Técnicas y Certificación ICONTEC NTC 4205. (2009). Unidades de mampostería de arcilla cocida. Ladrillos y bloques cerámicos. Parte 2: Mampostería no estructural. Bogotá, Colombia: ICONTEC.

[15] Sánchez Molina, J. y Ramírez Delgado, P. (2013). El Clúster de la cerámica del área metropolitana de Cúcuta (Trabajo de grado inédito). Universidad Francisco de Paula Santander. Cúcuta, Colombia. 